\title{
Thermodynamics and phase transition of a nonlinear electrodynamics black hole in a cavity
}

\author{
Peng Wang, Houwen $\mathrm{Wu}$ and Haitang Yang \\ Center for Theoretical Physics, College of Physics, Sichuan University, \\ Chengdu, Sichuan 610065, China \\ E-mail: pengw@scu.edu.cn, iverwu@scu.edu.cn, hyanga@scu.edu.cn
}

\begin{abstract}
We first discuss the thermodynamics of a Born-Infeld (BI) black hole enclosed in a finite spherical cavity. A canonical ensemble is considered, which means that the temperature and the charge on the wall of the cavity are fixed. After the free energy is obtained by computing the Euclidean action, it shows that the first law of thermodynamics is satisfied at the locally stationary points of the free energy. The phase structure and transition in various regions of the parameter space are then investigated. In the region where the BI electrodynamics has weak nonlinearities, Hawking-Page-like and van der Waals-like phase transitions occur, and a tricritical point appears. In the region where the BI electrodynamics has strong enough nonlinearities, only Hawking-Page-like phase transitions occur. The phase diagram of a BI black hole in a cavity can have dissimilarity from that of a BI black hole using asymptotically anti-de Sitter boundary conditions. The dissimilarity may stem from a lack of an appropriate reference state with the same charge and temperature for the BI-AdS black hole.
\end{abstract}

KEYwords: Black Holes, AdS-CFT Correspondence

ArXIV EPRINT: 1901.06216 


\section{Contents}

1 Introduction 1

2 Thermodynamics of Born-Infeld black hole in a cavity 2

2.1 Euclidean action 3

2.2 Thermodynamics 4

3 Phase structure of Born-Infeld black hole in a cavity 6

4 Discussion and conclusion $\quad 11$

\section{Introduction}

A Schwarzschild black hole in asymptotically flat space has negative specific heat and hence radiates more when it is smaller. To make this system thermally stable, appropriate boundary conditions must be imposed. One popular choice is putting the black hole in anti-de Sitter (AdS) space. The black hole becomes thermally stable since the AdS boundary acts as a reflecting wall. The thermodynamic properties of AdS black holes were first studied by Hawking and Page [1], who discovered the Hawking-Page phase transition between the thermal AdS space and the Schwarzschild-AdS black hole. Later, with the advent of the AdS/CFT correspondence [2-4], there has been much interest in studying the phase transition of AdS black holes [5-10]. However, it is not clear whether the duality between the black hole and a boundary field theory is independent of the details of the boundary conditions, or just the special result of the asymptotically AdS space. It is therefore interesting to investigate thermodynamics of black holes in the case of different boundary conditions.

Alternatively, one can place the black hole inside a cavity in asymptotically flat space, on the wall of which the metric is fixed. In [11], York showed that a Schwarzschild black hole in a cavity can be thermally stable and experiences a Hawking-Page-like transition to the thermal flat space as the temperature decreases. Later, the thermodynamics of a ReissnerNordstrom (RN) black hole in a cavity was discussed in a grand canonical ensemble [12] and a canonical ensemble $[13,14]$. Similar to a RN-AdS black hole, it was found that a Hawking-Page-like phase transition occurs in the grand canonical ensemble, and a van der Waals-like phase transition occurs in the canonical ensemble. The phase structure of several black brane systems in a cavity was investigated in a series of paper [15-20], where Hawking-Page-like or van der Waals-like phase transitions were always found except for some special cases. Including charged scalars, boson stars and hairy black holes in a cavity were considered in [21-24], which showed that the phase structure of the gravity system in a cavity is strikingly similar to that of holographic superconductors in the AdS gravity. The stabilities of solitons, stars and black holes in a cavity were also studied in [25-32]. It 
was found that the nonlinear dynamical evolution of a charged black hole in a cavity could end in a quasi-local hairy black hole. The thermodynamic behavior of de Sitter black holes in a cavity has been discussed in the extended phase space [33]. Recently, McGough, Mezei and Verlinde [34] proposed that the $T \bar{T}$ deformed $\mathrm{CFT}_{2}$ locates at the finite radial position of $\mathrm{AdS}_{3}$, which further motivates us to explore the properties of a black hole in a cavity.

Taking quantum contributions into account, nonlinear corrections are usually added to the Maxwell Lagrangian, which gives the nonlinear electrodynamics (NLED). Coupling NLED fields to gravity, various NLED charged black holes were derived and discussed in a number of papers [35-44]. It is interesting to note that some NLED black holes can be globally regular $[45,46]$. As pointed out in [47], a globally regular NLED black hole requires vanishing electric charge and a finite NLED Lagrangian (or in the FP dual theory). BornInfeld (BI) electrodynamics was first introduced to incorporates maximal electric fields and smooths divergences of the electrostatic self-energy of point charges [48]. Later, it is realized that BI electrodynamics can come from the low energy limit of string theory and encodes the low-energy dynamics of D-branes. The BI black hole solution was first obtained in $[49,50]$. The thermodynamic behavior and phase transition of BI black holes in various gravities were investigated in [51-67]. Especially, the thermodynamics of a 4D BI-AdS black hole was studied in [68-70], where a reentrant phase transition was always observed in a certain region of the parameter space.

In this paper, we first investigate the thermodynamic behavior of a BI black hole enclosed in a cavity. Then, we turn to study the phase structure and transition of the system. We find that Hawking-Page-like and van der Waals-like phase transitions can occur while there is no reentrant phase transition. The rest of this paper is organized as follows. In section 2, we compute the Euclidean action for a BI black hole in a cavity and discuss the thermodynamic properties of the system in the canonical ensemble. In section 3, we investigate the phase structure and transition of the system. The phase diagram is given in figure 1 , from which one can read the phase structure and transition.

\section{Thermodynamics of Born-Infeld black hole in a cavity}

In this section, we consider a BI black hole inside a cavity, on the boundary of which the temperature and charge are fixed. That said, the thermodynamics of the BI black hole is discussed in a canonical ensemble. On a $(3+1)$ dimensional spacetime manifold $\mathcal{M}$ with a time-like boundary $\partial \mathcal{M}$, the action of gravity coupled to a BI electromagnetic field $A_{\mu}$, is given by

$$
\mathcal{S}=\int_{\mathcal{M}} d^{4} x \sqrt{-g}\left[R+\frac{1}{a}\left(1-\sqrt{1+a F^{\mu \nu} F_{\mu \nu} / 2}\right)\right]+\mathcal{S}_{\text {surf }}
$$

where we take $16 \pi G=1$ for simplicity, the coupling parameter $a$ is related to the string tension $\alpha^{\prime}$ as $a=\left(2 \pi \alpha^{\prime}\right)^{2}>0$, and $S_{\text {surf }}$ are the surface terms on $\partial \mathcal{M}$. The surface terms of the action (2.1) are

$$
\mathcal{S}_{\text {surf }}=-2 \int_{\partial \mathcal{M}} d^{3} x \sqrt{-\gamma}\left(K-K_{0}\right)-\int_{\partial \mathcal{M}} d^{3} x \sqrt{-\gamma} \frac{A_{\mu} n_{\nu} F^{\mu \nu}}{\sqrt{1+a F^{\mu \nu} F_{\mu \nu} / 4}},
$$


where $K$ is the extrinsic curvature, $\gamma$ is the metric on the boundary, $K_{0}$ is a subtraction term to make the Gibbons-Hawking-York term vanish in flat spacetime, $n^{\mu}$ is the unit outward-pointing normal vector of $\partial \mathcal{M}$, and $F_{\mu \nu}=\partial_{\mu} A_{\nu}-\partial_{\nu} A_{\mu}$ is the BI electromagnetic field strength tensor. Note that the second term in $\mathcal{S}_{\text {surf }}$ is included to keep the charge fixed on $\partial \mathcal{M}$, instead of the potential [70]. The static spherically symmetric black hole solution of the action (2.1) was obtained in [49, 50]:

$$
\begin{aligned}
d s^{2} & =-f(r) d t^{2}+\frac{d r^{2}}{f(r)}+r^{2}\left(d \theta^{2}+\sin ^{2} \theta d \phi^{2}\right), \\
F_{r t}(r) & =A_{t}^{\prime}(r)=\frac{Q}{\sqrt{r^{4}+a Q^{2}}}
\end{aligned}
$$

where

$$
f(r)=1-\frac{M}{8 \pi r}-\frac{Q^{2}}{6 \sqrt{r^{4}+a Q^{2}}+6 r^{2}}+\frac{Q^{2}}{3 r^{2}}{ }_{2} F_{1}\left(\frac{1}{4}, \frac{1}{2}, \frac{5}{4} ;-\frac{a Q^{2}}{r^{4}}\right),
$$

and $M$ and $Q$ are the mass and charge of the black hole [70], respectively. On the boundary of the cavity $\partial \mathcal{M}$ at $r=r_{B}$, the gauge potential measured on $\partial \mathcal{M}$ with respect to the horizon at $r=r_{+}$is

$$
\Phi=\frac{4 \pi}{\sqrt{f\left(r_{B}\right)}} \int_{r_{+}}^{r_{B}} A_{t}^{\prime}(r) d r=\frac{4 \pi A_{t}\left(r_{B}\right)}{\sqrt{f\left(r_{B}\right)}}
$$

where the blueshift factor $1 / \sqrt{f\left(r_{B}\right)}$ relates $A_{t}$ to the proper orthonormal frame component of the potential one-form [12], and we fix the gauge field $A_{t}(r)$ at the horizon to be zero.

\subsection{Euclidean action}

In the semiclassical approximation, one can relate the on-shell Euclidean action to the thermal partition function:

$$
Z \simeq e^{-\mathcal{S}^{E}}
$$

where $\mathcal{S}^{E}$ is the Euclidean continuation of the action $\mathcal{S}: \mathcal{S}^{E}=i \mathcal{S}$. The Euclidean time $\tau$ is obtained from Lorentzian time $t$ by the analytic continuation $\tau=i t$. From $A_{\tau} d \tau=A_{t} d t$, it follows that

$$
A_{\tau}=-i A_{t}
$$

Since the temperature $T$ is fixed on the boundary of the cavity, we can impose the boundary condition at $r=r_{B}$ in terms of the reciprocal temperature:

$$
\int \sqrt{f\left(r_{B}\right)} d \tau=T^{-1}
$$

which identifies the Euclidean time $\tau$ as $\tau \sim \tau+\frac{1}{T \sqrt{f\left(r_{B}\right)}}$, and hence the period of $\tau$ is $\frac{1}{T \sqrt{f\left(r_{B}\right)}}$. 
For the black hole solution (2.3) in a cavity, one can evaluate the Euclidean action by integrating over angles and performing the integration by parts:

$$
\begin{aligned}
\mathcal{S}^{E}= & -\frac{8 \pi}{T \sqrt{f\left(r_{B}\right)}} \int_{r_{+}}^{r_{B}} d r\left[f(r)+1+r f^{\prime}(r)\right]+\frac{16 \pi r_{B}}{T} \\
& -16 \pi^{2} r_{+}^{2}-\frac{4 \pi}{T \sqrt{f\left(r_{B}\right)}} \int_{r_{+}}^{r_{B}} d r \frac{r^{2}\left(1-\sqrt{1+a A_{\tau}^{\prime 2}(r)}\right)}{a}+\frac{4 \pi i A_{\tau}\left(r_{B}\right) Q}{T \sqrt{f\left(r_{B}\right)}} .
\end{aligned}
$$

After eqs. (2.3) and (2.4) are plugged into eq. (2.9), a straightforward calculation gives

$$
\mathcal{S}^{E}=\frac{16 \pi r_{B}}{T}\left[1-\sqrt{f\left(r_{B}\right)}\right]-S,
$$

where $S=16 \pi^{2} r_{+}^{2}$ is the entropy of the black hole. In the limit of $r_{B} \rightarrow \infty$, the Euclidean action then reduces to

$$
\mathcal{S}^{E}=\frac{1}{T}(M-T S)
$$

where we use

$$
f\left(r_{B}\right)=1-\frac{M}{8 \pi r_{B}}+\frac{Q^{2}}{4 r_{B}^{2}}+\mathcal{O}\left(r_{B}^{-4}\right) .
$$

\subsection{Thermodynamics}

Various thermodynamic quantities can be derived from the Euclidean action (2.10), which is related to the free energy $F$ in the semiclassical approximation by

$$
F=-T \ln Z=T \mathcal{S}^{E} .
$$

From eqs. (2.4) and (2.10), one finds that the free energy $F$ is a function of the temperature $T$, the charge $Q$, the cavity radius $r_{B}$ and the horizon radius $r_{+}$:

$$
F=F\left(r_{+} ; T, Q, r_{B}\right)
$$

where $T, Q$ and $r_{B}$ are parameters of the canonical ensemble. The only variable $r_{+}$can be determined by extremizing the free energy $F\left(r_{+} ; T, Q, r_{B}\right)$ with respect to $r_{+}$:

$$
\frac{d F\left(r_{+} ; T, Q, r_{B}\right)}{d r_{+}}=0 \Longrightarrow-\frac{d\left[r_{B} f\left(r_{B}\right)\right] / d r_{+}}{2 \sqrt{f\left(r_{B}\right)}}=2 \pi r_{+} T \Longrightarrow f^{\prime}\left(r_{+}\right)=4 \pi T \sqrt{f\left(r_{B}\right)},
$$

where we use $d\left[r_{B} f\left(r_{B}\right)\right] / d r_{+}=-r_{+} f^{\prime}\left(r_{+}\right)$. That said, the solution $r_{+}=r_{+}\left(T, Q, r_{B}\right)$ of eq. (2.15) corresponds to a locally stationary point of $F\left(r_{+} ; T, Q, r_{B}\right)$. It is interesting to note that eq. (2.15) can be written as

$$
T=\frac{T_{h}}{\sqrt{f\left(r_{B}\right)}}
$$

where

$$
T_{h}=\frac{f^{\prime}\left(r_{+}\right)}{4 \pi}
$$


is the Hawking temperature of the black hole. The temperature $T$ on $\partial \mathcal{M}$ is thus blueshifted from $T_{h}$, which is measured at infinity.

After obtaining $r_{+}=r_{+}\left(T, Q, r_{B}\right)$, we can evaluate $F\left(r_{+} ; T, Q, r_{B}\right)$ at the locally stationary point $r_{+}=r_{+}\left(T, Q, r_{B}\right)$ :

$$
F\left(T, Q, r_{B}\right) \equiv F\left(r_{+}\left(T, Q, r_{B}\right) ; T, Q, r_{B}\right) .
$$

For later convenience, we shall suppress $T, Q$ and $r_{B}$ in $F\left(r_{+} ; T, Q, r_{B}\right)$ and $F\left(T, Q, r_{B}\right)$ and denote $F\left(r_{+} ; T, Q, r_{B}\right)$ and $F\left(T, Q, r_{B}\right)$ as $F\left(r_{+}\right)$and $F$, respectively. The thermal energy is

$$
E=-T^{2} \frac{\partial(F / T)}{\partial T}=16 \pi r_{B}\left[1-\sqrt{f\left(r_{B}\right)}\right]
$$

Using eq. (2.4), we can express the ADM mass of the black hole $M$ in terms of $E$ and $Q$ :

$$
M=E-\frac{E^{2}}{32 \pi r_{B}}-8 \pi r_{B}\left[\frac{Q^{2}}{6 \sqrt{r_{B}^{4}+a Q^{2}}+6 r_{B}^{2}}-\frac{Q^{2}}{3 r_{B}^{2}}{ }_{2} F_{1}\left(\frac{1}{4}, \frac{1}{2}, \frac{5}{4} ;-\frac{a Q^{2}}{r_{B}^{4}}\right)\right],
$$

where the second and third terms on left-hand side can be interpreted as the gravitational and electrostatic binding energies, respectively. Using eq. (2.4), we can express the thermal energy $E$ in terms of the entropy $S$, the charge $Q$ and the cavity radius $r_{B}$. Differentiating $E$ with respect to $S$ and $Q$, respectively, gives

$$
\begin{aligned}
& \frac{\partial E}{\partial S}=-\frac{d\left[r_{B} f\left(r_{B}\right)\right] / d r_{+}}{4 \pi r_{+} \sqrt{f\left(r_{B}\right)}}=T, \\
& \frac{\partial E}{\partial Q}=-\frac{8 \pi r_{B}}{\sqrt{f\left(r_{B}\right)}} \frac{\partial f\left(r_{B}\right)}{\partial Q}=\Phi .
\end{aligned}
$$

From the energy $E$, we can define a thermodynamic surface pressure by

$$
\lambda \equiv-\frac{\partial E}{\partial\left(4 \pi r_{B}^{2}\right)}=\frac{\left(1-\sqrt{f\left(r_{B}\right)}\right)^{2}}{r_{B} \sqrt{f\left(r_{B}\right)}}+\frac{r_{B}^{2}-\sqrt{r_{B}^{4}+a Q^{2}}}{2 a r_{B} \sqrt{f\left(r_{B}\right)}} .
$$

From eqs. (2.21) and (2.22), the first law of thermodynamics can be established:

$$
d E=T d S+\Phi d Q-\lambda d A
$$

where $A \equiv 4 \pi r_{B}^{2}$ is the surface area of the cavity.

To obtain the proper Smarr relation for a BI black hole in a cavity, we need to consider the dimensionful couplings $a$ in the action (2.1), where one has $[a]=[R]^{-1}=L^{2}$. Since the dimensional analysis gives that $[Q]=[E]=L,[T]=[\lambda]=L^{-1}$ and $[A]=[S]=L^{2}$, the thermal energy $E$, as a function of $S, Q, A$ and $a$, satisfies

$$
E\left(\alpha^{2} S, \alpha Q, \alpha^{2} A, \alpha^{2} a\right)=\alpha E(S, Q, A, a),
$$

where $\alpha$ is a positive number. So the Euler's theorem leads to the Smarr relation:

$$
\begin{aligned}
E & =2\left(S \frac{\partial E}{\partial S}+A \frac{\partial E}{\partial A}+a \frac{\partial E}{\partial a}\right)+Q \frac{\partial E}{\partial Q} \\
& =2(T S-\lambda A+a \mathcal{A})+Q \Phi,
\end{aligned}
$$


where we introduce the conjugates $\mathcal{A}$ associated with $a$ :

$$
\mathcal{A}=\frac{\partial E}{\partial a}
$$

and use eqs. (2.21) and (2.22). In the limit of $r_{B} \rightarrow \infty$, eq. (2.25) gives the Smarr relation for an asymptotically flat BI black hole:

$$
M=2(T S+a \mathcal{A})+Q \Phi \text { with } \mathcal{A}=\frac{\partial M}{\partial a} .
$$

The Smarr relation for a BI-AdS black hole was given in eq. (5.14) in [68], which agrees with eq. (2.27) in the limit of zero cosmological constant. ${ }^{1}$

To find the global minimum of $F\left(r_{+}\right)$over the space of the variable $r_{+}$with fixed values of $T, Q$ and $r_{B}$, we also need to consider the values of $F\left(r_{+}\right)$at the edges of the space of $r_{+}$. In fact, the physical space of $r_{+}$is constrained by

$$
r_{e} \leq r_{+} \leq r_{B},
$$

where $r_{e}$ is the horizon radius of the extremal black hole. If there exists no extremal black hole solution, one can simply set $r_{e}=0$. For simplicity, the global minimum of $F\left(r_{+}\right)$at the edges is dubbed "edge state (ES)" in our paper.

\section{Phase structure of Born-Infeld black hole in a cavity}

From eq. (2.4), one can express $f(r)$ in terms of the horizon radius $r_{+}$:

$$
\begin{aligned}
f(r)= & 1-\frac{r_{+}}{r}+\frac{r_{+}}{r} \frac{Q^{2}}{6 \sqrt{r_{+}^{4}+a Q^{2}}+6 r_{+}^{2}}-\frac{Q^{2}}{3 r r_{+}}{ }_{2} F_{1}\left(\frac{1}{4}, \frac{1}{2}, \frac{5}{4} ;-\frac{a Q^{2}}{r_{+}^{4}}\right) \\
& -\frac{Q^{2}}{6 \sqrt{r^{4}+a Q^{2}}+6 r^{2}}+\frac{Q^{2}}{3 r^{2}}{ }_{2} F_{1}\left(\frac{1}{4}, \frac{1}{2}, \frac{5}{4} ;-\frac{a Q^{2}}{r^{4}}\right) .
\end{aligned}
$$

It is convenient to express quantities in units of $r_{B}$ :

$$
x \equiv \frac{r_{+}}{r_{B}}, \tilde{Q} \equiv \frac{Q}{r_{B}}, \tilde{a} \equiv \frac{a}{r_{B}^{2}}, \tilde{T} \equiv r_{B} T, \tilde{F}(x)=\frac{F\left(r_{+}\right)}{16 \pi r_{B}} \text { and } \tilde{F}=\frac{F}{16 \pi r_{B}} .
$$

We then use eqs. (2.10) and (3.1) to find the free energy as a function of $x$ :

$$
\tilde{F}(x)=1-\sqrt{f(x)}-\pi x^{2} \tilde{T},
$$

where

$$
\begin{aligned}
f(x)= & 1-x+\frac{x \tilde{Q}^{2}}{6 \sqrt{x^{4}+\tilde{a} \tilde{Q}^{2}}+6 x^{2}}-\frac{\tilde{Q}^{2}}{3 x}{ }_{2} F_{1}\left(\frac{1}{4}, \frac{1}{2}, \frac{5}{4} ;-\frac{\tilde{a} \tilde{Q}^{2}}{x^{4}}\right) \\
& -\frac{\tilde{Q}^{2}}{6 \sqrt{1+\tilde{a} \tilde{Q}^{2}}+6}+\frac{\tilde{Q}^{2}}{3}{ }_{2} F_{1}\left(\frac{1}{4}, \frac{1}{2}, \frac{5}{4} ;-\tilde{a} \tilde{Q}^{2}\right) .
\end{aligned}
$$

\footnotetext{
${ }^{1}$ In [68], their $b$ is our $\frac{1}{\sqrt{a}}$. So $-b \mathcal{B}$ in eq. (5.14) becomes

$$
-b \mathcal{B}=-b \frac{\partial M}{\partial b}=2 a \frac{\partial M}{\partial a}=2 a \mathcal{A} .
$$
}


The Hawking temperature of the BI black hole can be calculated from eq. (2.17):

$$
\tilde{T}_{h} \equiv r_{B} T_{h}=\frac{1}{4 \pi x}\left(1-\frac{1}{2} \frac{\tilde{Q}^{2}}{x^{2}+\sqrt{x^{4}+\tilde{a} \tilde{Q}^{2}}}\right)
$$

which relates to $\tilde{T}$ as

$$
\tilde{T}=\frac{\tilde{T}_{h}}{\sqrt{f(x)}} .
$$

As shown in [38], there are two types of BI black holes depending on the minimum value of $\tilde{T}_{h}$ :

- RN type: $4 \tilde{a} \leq \tilde{Q}^{2} \leq 4(1+\tilde{a})$. This type of BI black holes can have extremal black hole solutions like RN black holes. For $\tilde{T}_{h}=0$, there is one single solution $x=x_{e} \equiv \frac{1}{2} \sqrt{\tilde{Q}^{2}-4 \tilde{a}}$, where $x_{e} r_{B}$ is the horizon radius of the extremal BI black hole. In this case, we must have $x_{e} \leq x \leq 1$. Note that $x_{e} \leq 1$ puts an upper bound on $\tilde{Q}^{2}: \tilde{Q}^{2} \leq 4(1+\tilde{a})$. Another way to understand this upper bound is that $f(x)$ is always negative when $\tilde{Q}^{2}>4(1+\tilde{a})$, and hence $\tilde{F}(x)$ is not a real-valued function for any $x$. We have an extremal BI black hole when $x=x_{e}$, and the horizon merges with the boundary when $x=1$.

- Schwarzschild-like type: $\tilde{Q}^{2}<4 \tilde{a}$. This type of BI black holes have only one horizon like Schwarzschild black holes. The Hawking temperature $\tilde{T}_{h}$ has a positive minimum value and goes to $+\infty$ as $x \rightarrow 0$. In this case, we can have $0 \leq x \leq 1$, over which $\tilde{F}(x)$ is well-defined. It can show that $\tilde{T}_{h} / \sqrt{f(x)}$ has a minimum value of $\tilde{T}_{\text {min }}>0$ over $0 \leq x \leq 1$. Eq. (3.6) implies that the locally stationary points of $\tilde{F}(x)$ only exist for $\tilde{T} \geq \tilde{T}_{\min }$. When $x=0$, one finds that eq. (3.1) becomes

$$
f(r)=1-\frac{Q^{3 / 2} \Gamma(1 / 4) \Gamma(5 / 4)}{3 a^{1 / 4} \sqrt{\pi} r}-\frac{Q^{2}}{6 \sqrt{r^{4}+a Q^{2}}+6 r^{2}}+\frac{Q^{2}}{3 r^{2}}{ }_{2} F_{1}\left(\frac{1}{4}, \frac{1}{2}, \frac{5}{4} ;-\frac{a Q^{2}}{r^{4}}\right) .
$$

When $Q=0$, the edge state at $x=0$ is just the thermal flat space. For $Q>0$, we have

$$
f(r)=1-\frac{Q}{2 \sqrt{a}}+\mathcal{O}(r) \text { and } R=\frac{Q}{\sqrt{a} r^{2}}-\frac{2}{a}+\mathcal{O}(r),
$$

where $R$ is the Ricci scalar. So the metric has a physical singularity at $r=0$ although $f(0)$ is finite. Since $f(r)>0$, there exists no horizon. The edge state with $Q>0$ at $x=0$ is thus a naked singularity.

To find the phase structure and transition of a BI black hole in a cavity, we need to analyze the locally stationary points of $\tilde{F}(x)$ and find the global minimum value of $\tilde{F}(x)$. In fact, the locally stationary points of $\tilde{F}(x)$ can be determined by solving eq. (3.6) for $x$ in terms of $\tilde{T}$ and $\tilde{Q}$. The solution $x(\tilde{T}, \tilde{Q})$ is often a multivalued function, each branch of which corresponds to a family of BI black hole solutions. In [70], it showed that a thermally stable branch has $\partial x(\tilde{T}, \tilde{Q}) / \partial \tilde{T}>0$ while an unstable one has $\partial x(\tilde{T}, \tilde{Q}) / \partial \tilde{T}<0$. We find 

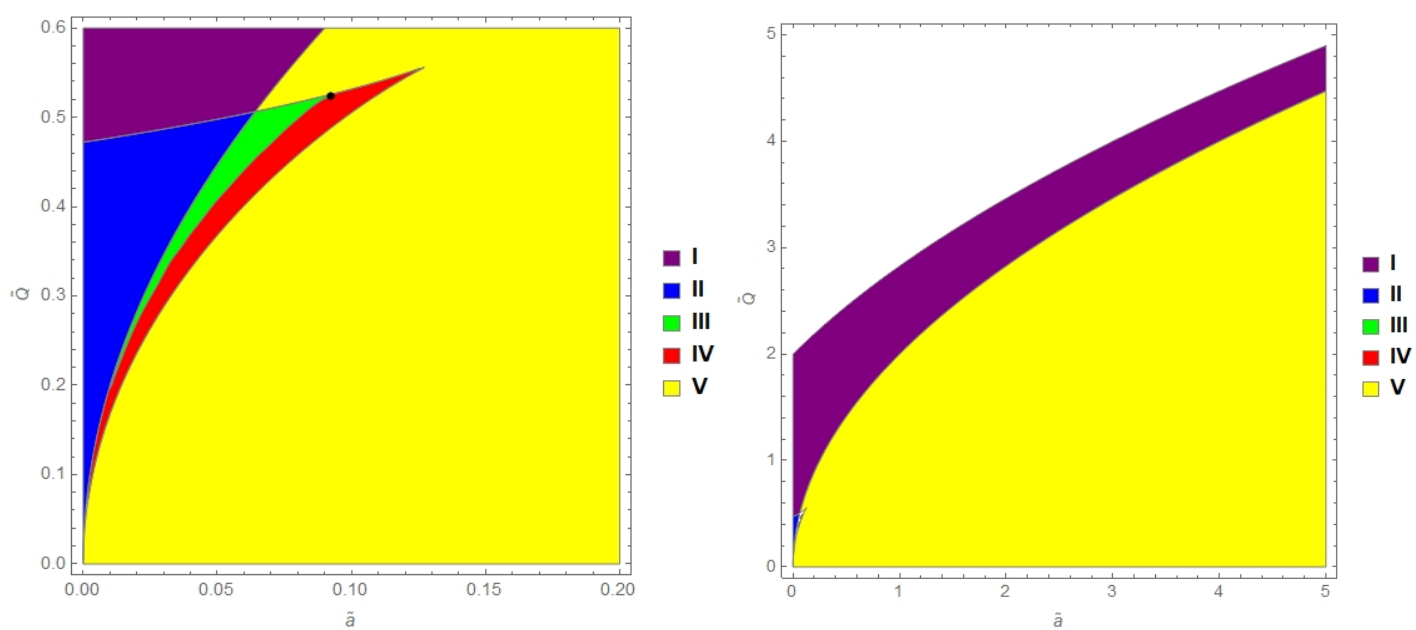

Figure 1. The five regions in the $\tilde{a}-\tilde{Q}$ phase space of a BI black hole (BH) in a cavity, each of which possesses distinct behavior of the phase structure and transition. Varying the temperature, a van der Waals-like LBH/SBH phase transition occurs in Regions II and III while a Hawking-Page-like ES/BH phase transition occurs in Regions III, IV and V. There is only one phase in Regions I. The critical line consists of $\tilde{Q}_{12}(\tilde{a})$ and $\tilde{Q}_{35}(\tilde{a})$, where $\tilde{Q}_{i j}(\tilde{a})$ is the boundary between Region $i$ and Region $j$. The critical line terminates at the black dot, which is at $\left\{\tilde{a}_{c}, \tilde{Q}_{c}\right\} \approx\{0.092,0.524\}$.

that there are five regions in the $\tilde{a}-\tilde{Q}$ phase space, in each of which the BI black hole has distinct behavior of the branches of $x(\tilde{T}, \tilde{Q})$ and phase structure. These five regions are mapped in figure 1 . We plot $x(\tilde{T}, \tilde{Q})$ against $\tilde{T}$ for various values of $\tilde{Q}$ with $\tilde{a}=0.05$ in figure 2 , which shows the general behavior of $x(\tilde{T}, \tilde{Q})$ in these five regions. In what follows, we discuss the phase structure and transition of the BI black hole in the five regions:

- Region I: As shown in figure 2, there is only one branch for $x(\tilde{T}, \tilde{Q})$, on which the BI black hole is thermally stable. The BI black hole in this region satisfies $\tilde{Q} \geq 4 \tilde{a}$ and hence is RN type. For the BI black hole with $\tilde{a}=0.01$ and $\tilde{Q}=0.6$ in this region, we plot the free energy $\tilde{F}(x)$ in figure $3 \mathrm{a}$, which shows that the endpoints always have higher free energy, and the local minimum of $\tilde{F}(x)$ is the global minimum. There is only one phase in this region.

- Region II: The BI black hole in this region is also RN type. As shown in figure 2, there are three branches for $x(\tilde{T}, \tilde{Q})$, which are denoted by Small BH (green), Large BH (blue) and Intermediate BH (brown). Both the Small BH and Large BH branches are thermally stable. For the BI black hole with $\tilde{a}=0.01$ and $\tilde{Q}=0.3$ in this region, we plot the free energy $\tilde{F}(x)$ in figure 3 b, which shows that the endpoints always have higher free energy than one of the locally minima. So $\tilde{F}(x)$ has the global minimum at Small BH for small enough $\tilde{T}$ and Large BH for large enough $\tilde{T}$, respectively. The free energies of the three branches are plotted in the right panel of figure $3 \mathrm{~b}$, which shows that there is a first-order phase transition between Small BH and Large BH.

- Region III: The BI black hole in this region is Schwarzschild-like type. For $\tilde{T}<\tilde{T}_{\min }$, $\tilde{F}(x)$ is a strictly increasing function (see $\tilde{T}=0.10$ in the left panel of figure 4 ), and 


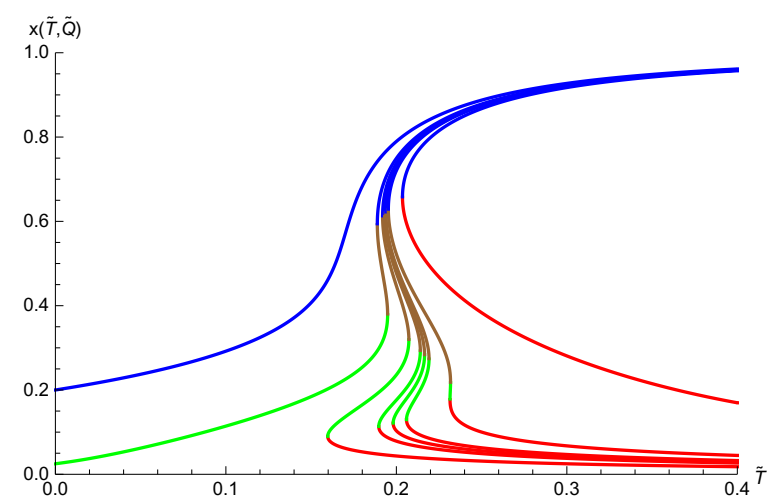

Figure 2. Plots of $x(\tilde{T}, \tilde{Q})$ against $\tilde{T}$ for various values of $\tilde{Q}$ with $\tilde{a}=0.05$, where $x(\tilde{T}, \tilde{Q})$ is the locally stationary point of $\tilde{F}(x)$. From left to right, $\tilde{Q}=0.600$ (Region I), 0.450 (Region II), 0.415 (Region III), 0.400 (Region IV), 0.395 (Region IV), 0.390 (Region IV), 0.370 (Region IV) and 0.200 (Region V). Since thermally stable phases have $\partial x / \partial \tilde{T}>0$, the BI BHs on blue and green segments of the curves are thermally stable. We denote the BI BHs on blue segments by Large BH (or BH if there is no green segment on the curve) and these on green segments by Small BH. The BI BHs on red and brown segments are denoted by Intermediate $\mathrm{BH}$, which are thermally unstable.

hence $\tilde{F}(x)$ has the global minimum at $x=0$. For $\tilde{T} \geq \tilde{T}_{\min }$, as shown in figure 2 , there are four branches for $x(\tilde{T}, \tilde{Q})$, which are denoted by Small BH (green), Large $\mathrm{BH}$ (blue) and Intermediate BH (brown and red). Both the Small BH and Large $\mathrm{BH}$ branches are thermally stable. The free energies of the four branches and the edge state are plotted in the right panel of figure 4 . As $\tilde{T}$ increases from $\tilde{T}_{\min }$, the free energy of Small BH decreases while $\tilde{F}(0)$ is constant. They cross each other at some point, where a first-order phase transition occurs, and Small BH then becomes globally stable after the crossing. Further increasing $\tilde{T}$, Large BH appears, and its free energy decreases more rapidly than that of Small BH. So they cross each other at some point, where another first-order transition occurs, and Large BH then becomes the globally stable phase.

- Region IV: The BI black hole in this region is also Schwarzschild-like type. As in Region III, the edge state at $x=0$ is globally stable for $\tilde{T}<\tilde{T}_{\min }$. For $\tilde{T} \geq \tilde{T}_{\min }$, there are also four branches for $x(\tilde{T}, \tilde{Q})$, which are denoted by Small BH (green), Large BH (blue) and Intermediate BH (brown and red). The free energies of the four branches and the edge state are plotted in figure 5a. Unlike Region III, the temperature at which Small BH appears is too high, such that $\tilde{F}(0)$ does not cross the free energy of Small BH or $\tilde{F}(0)$ crosses the free energy of Large BH before it crosses that of Small BH. Hence as $\tilde{T}$ increases, there is only one first-order transition from the edge state to Large $\mathrm{BH}$ at some temperature, where the free energy of Large $\mathrm{BH}$ and $\tilde{F}(0)$ cross each other.

- Region V: The BI black hole in this region is also Schwarzschild-like type. As in Regions III and IV, the edge state at $x=0$ is globally stable for $\tilde{T}<\tilde{T}_{\min }$. However for $\tilde{T} \geq \tilde{T}_{\min }$, there are two branches for $x(\tilde{T}, \tilde{Q})$, which are denoted by BH (green) 

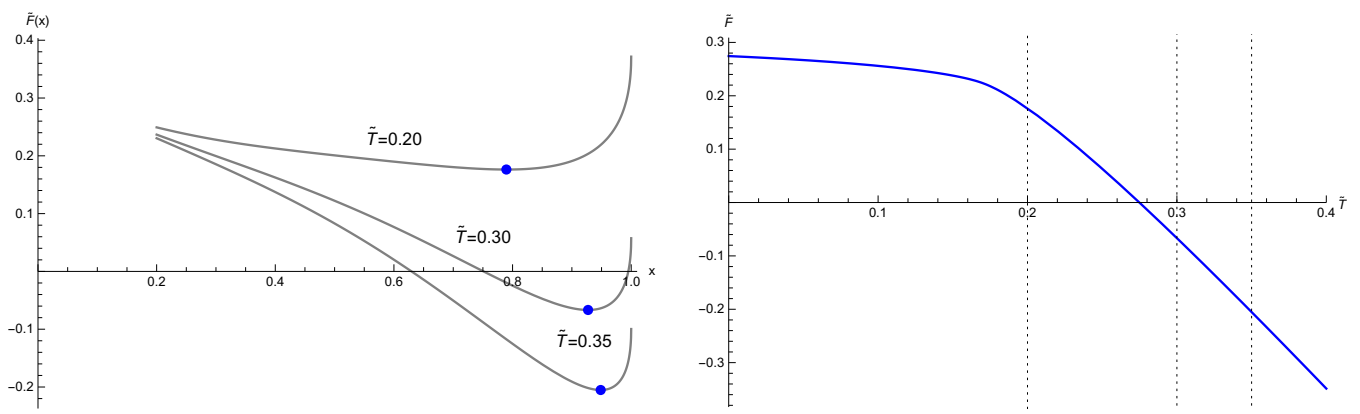

(a) Region I: $\tilde{a}=0.01$ and $\tilde{Q}=0.6$. There is no phase transition.
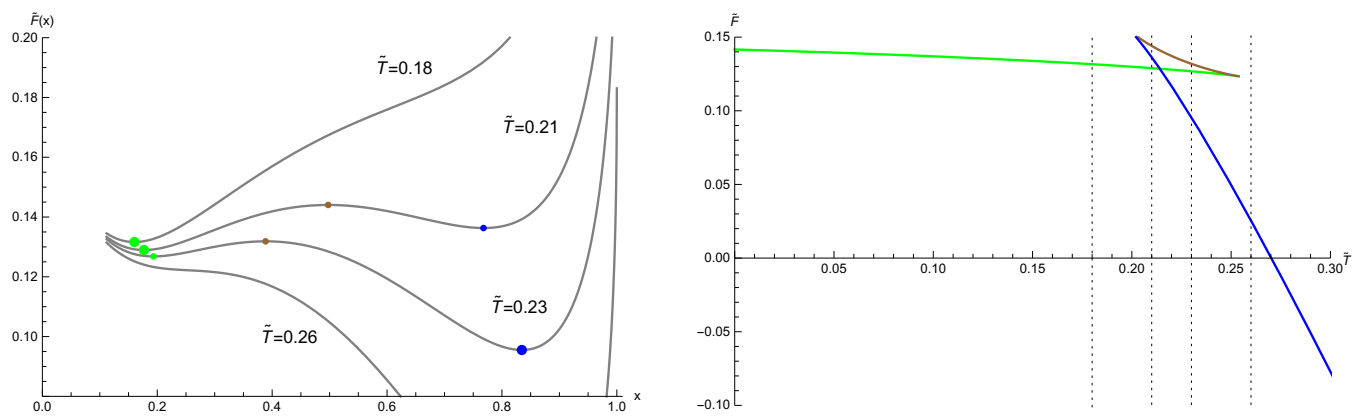

(b) Region II: $\tilde{a}=0.01$ and $\tilde{Q}=0.3$. There is a first-order phase transition between Small $\mathrm{BH}$ and Large BH.

Figure 3. Plots of the free energies $\tilde{F}(x)$ and $\tilde{F}$ against $\tilde{T}$ for the BI BHs in Regions I and II. The BI BHs in these regions are RN type. The BI BHs on the blue and green branches (dots) are thermally stable. Left Panels: $\tilde{F}(x)$ is plotted for various values of $\tilde{T}$, which are depicted by the vertical black dotted lines in the right panels. The locally stationary points of $\tilde{F}(x)$ are marked with colored dots, the colors of which correspond to these of segments in figure 2. Larger dots represent global minima of $\tilde{F}(x)$, which are globally stable. Right Panels: the values of $\tilde{F}(x)$ evaluated at the locally stationary points are plotted against $\tilde{T}$. Their colors match these in the left panels and figure 2, which means that blue/green branches are Large/Small BH, and the brown branch is Intermediate $\mathrm{BH}$.

and Intermediate $\mathrm{BH}$ (red). The $\mathrm{BH}$ branch is thermally stable. The free energies of the two branches and the edge state are plotted in figure 5b, which shows that there is a first-order transition from the edge state to Large $\mathrm{BH}$ as $\tilde{T}$ increases.

In figure 1 , the boundary between the region in which $x(\tilde{T}, \tilde{Q})$ has $n$ branches and that in which $x(\tilde{T}, \tilde{Q})$ has $n+2$ branches is the critical line. There are 3 such boundaries in figure 1, i.e., $\tilde{Q}_{12}(\tilde{a}), \tilde{Q}_{35}(\tilde{a})$ and $\tilde{Q}_{45}(\tilde{a})$, where $\tilde{Q}_{i j}(\tilde{a})$ is the boundary between Region $i$ and Region $j$. However, figure 5 shows that $\tilde{Q}_{45}(\tilde{a})$ is not physical since it does not globally minimize the free energy. Thus physical critical line only consists of $\tilde{Q}_{12}(\tilde{a})$ and $\tilde{Q}_{35}(\tilde{a})$, which terminates at $\left\{\tilde{a}_{c}, \tilde{Q}_{c}\right\} \simeq\{0.092,0.524\}$. The line $\tilde{Q}_{12}(\tilde{a})$ is reminiscent of RN black holes.

The phase diagram with $\tilde{a}=0.05$ in the $\tilde{Q}-\tilde{T}$ phase space is displayed in the left panel of figure 6 . There is a LBH/SBH first-order phase transition for some range of $\tilde{Q}$, a Hawking-Page-like ES/SBH first-order phase transition for some smaller range of $\tilde{Q}$ and 

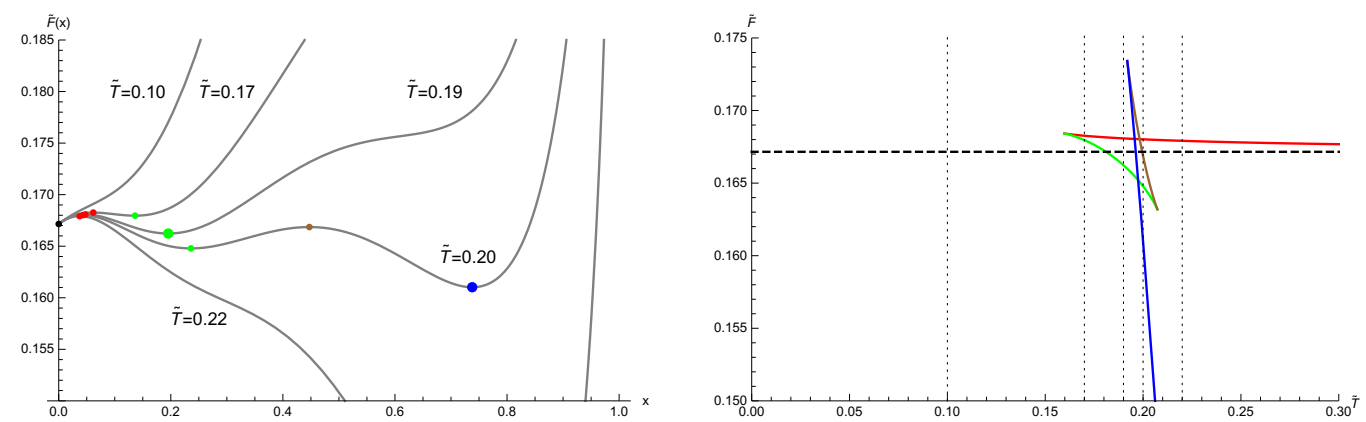

Figure 4. Region III: $\tilde{a}=0.05$ and $\tilde{Q}=0.415$. The BI BHs in this region are Schwarzschildlike type, which implies that $x$ can go to 0 . Left Panel: the locally stationary points of $\tilde{F}(x)$ are represented with colored dots, and $\tilde{F}(0)$ is marked with the black dot. Larger colored dots represent global minimums of $\tilde{F}(x)$. If there is no larger colored dot for some $\tilde{T}, \tilde{F}(x)$ has the global minima at $x=0$. Right Panel: the colored branches are the values of $\tilde{F}(x)$ evaluated at the locally stationary points as functions of $\tilde{T}$. Specifically, the blue/green branches are Large/Small $\mathrm{BH}$, and the brown/red branches are Intermediate BH. The horizontal black dashed line is $\tilde{F}(0)$. As $\tilde{T}$ increases from zero, a first-order phase transition from the edge state to Small BH occurs at some $\tilde{T}$. Further increasing $\tilde{T}$, there would be another first-order phase transition from Small BH to Large $\mathrm{BH}$.

another Hawking-Page-like ES/BH first-order phase transition for some larger range of $\tilde{Q}$. The LBH/SBH phase transition line is a van der Waals-like phase transition and hence terminates at the critical point, represented by the red dot. These three first-order phase transition lines merge together at the tricritical point, marked by the green dot. The phase diagram with $\tilde{a}=0.15$ in the $\tilde{Q}-\tilde{T}$ phase space is plotted in the right panel of figure 6 , which is simpler than that with $\tilde{a}=0.05$. Since $\tilde{a}=0.15>\tilde{a}_{c}$, there is no critical point or tricritical point in this phase diagram. There are two phases, namely the edge state and BH, which are separated by a Hawking-Page-like first-order phase transition line.

\section{Discussion and conclusion}

In the first part of this paper, we calculated the Euclidean action of a BI black hole in a finite spherical cavity and investigated the corresponding thermodynamic behavior in a canonical ensemble. Specifically, the Euclidean action of the system was given by eq. (2.10), which could be related to the free energy. It was then demonstrated that the first law of thermodynamics and the Smarr relation were satisfied at the locally stationary points of the free energy. It also showed that the local minimum of the free energy corresponds to the locally stable phase of the system. To determine the globally stable phase, the edge states are needed to be considered as well.

In the second part, we examined the phase structure and transition of a BI black hole in a cavity. In figure 1 , we mapped five regions in the $\tilde{a}-\tilde{Q}$ phase space, each of which has different phase behavior. Regions I and II are reminiscent of RN black holes, where there exist extremal black hole solutions. In these two regions, the global minimum of the free energy is always at one of the locally stationary points. There is only one branch of black 

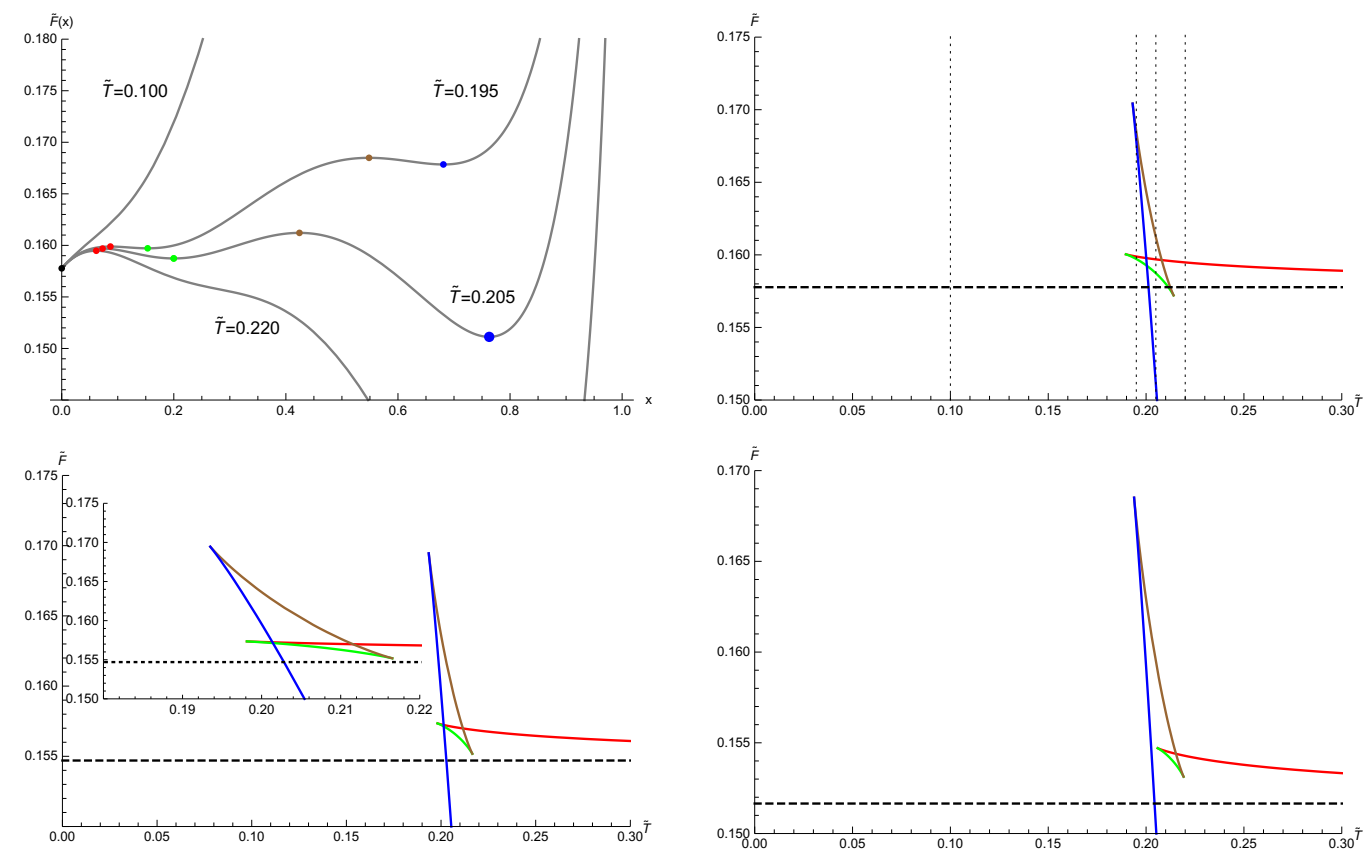

(a) Region IV: $\tilde{a}=0.05$. Top Panels: $\tilde{Q}=0.400$. Bottom-Left Panel: $\tilde{Q}=0.395$. Bottom-Right Panel: $\tilde{Q}=0.390$.
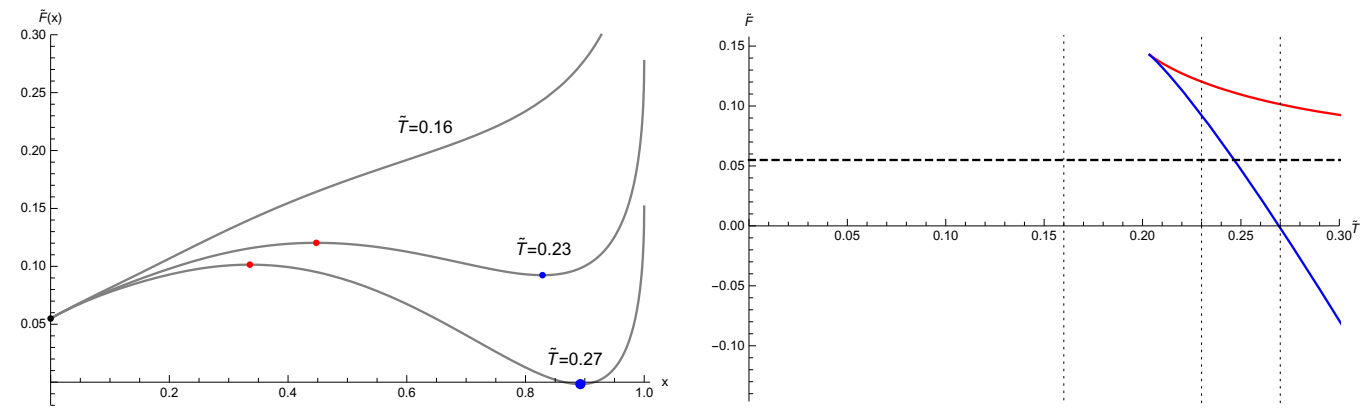

(b) Region $\mathrm{V}: \tilde{a}=0.05$ and $\tilde{Q}=0.4$.

Figure 5. Plots of the free energies $\tilde{F}(x)$ and $\tilde{F}$ against $\tilde{T}$ for the BI BHs in Regions IV and $\mathrm{V}$, which are Schwarzschild-like type. The blue/green branches are Large/Small BH, and the brown/red branches are Intermediate BH. The horizontal black dashed line is $\tilde{F}(0)$. As $\tilde{T}$ increases from zero, there is always a single first-order phase transition from the edge state to Large $\mathrm{BH}$ occurring at some temperature.

hole solutions in Region I, while in Region II, there is a band of temperatures where three branches coexist, and a first-order $\mathrm{LBH} / \mathrm{SBH}$ phase transition occurs. In Regions III, IV and $\mathrm{V}$, for low temperatures, the global minimum of the free energy is at $x=0$, which describes a naked singularity. For high enough temperatures, the global minimum is at one of the locally stationary points, and hence there is a first-order phase transition from the edge state at $x=0$ to the black hole phase as the temperature increases. In Region III, further increasing the temperature will lead to another first-order phase transition from Small BH to Large BH. The phase diagrams with $\tilde{a}=0.05$ and 0.15 in the $\tilde{Q}-\tilde{T}$ phase space were plotted in figure 6 , respectively. For $\tilde{a}=0.05$, there are three first-order phase 

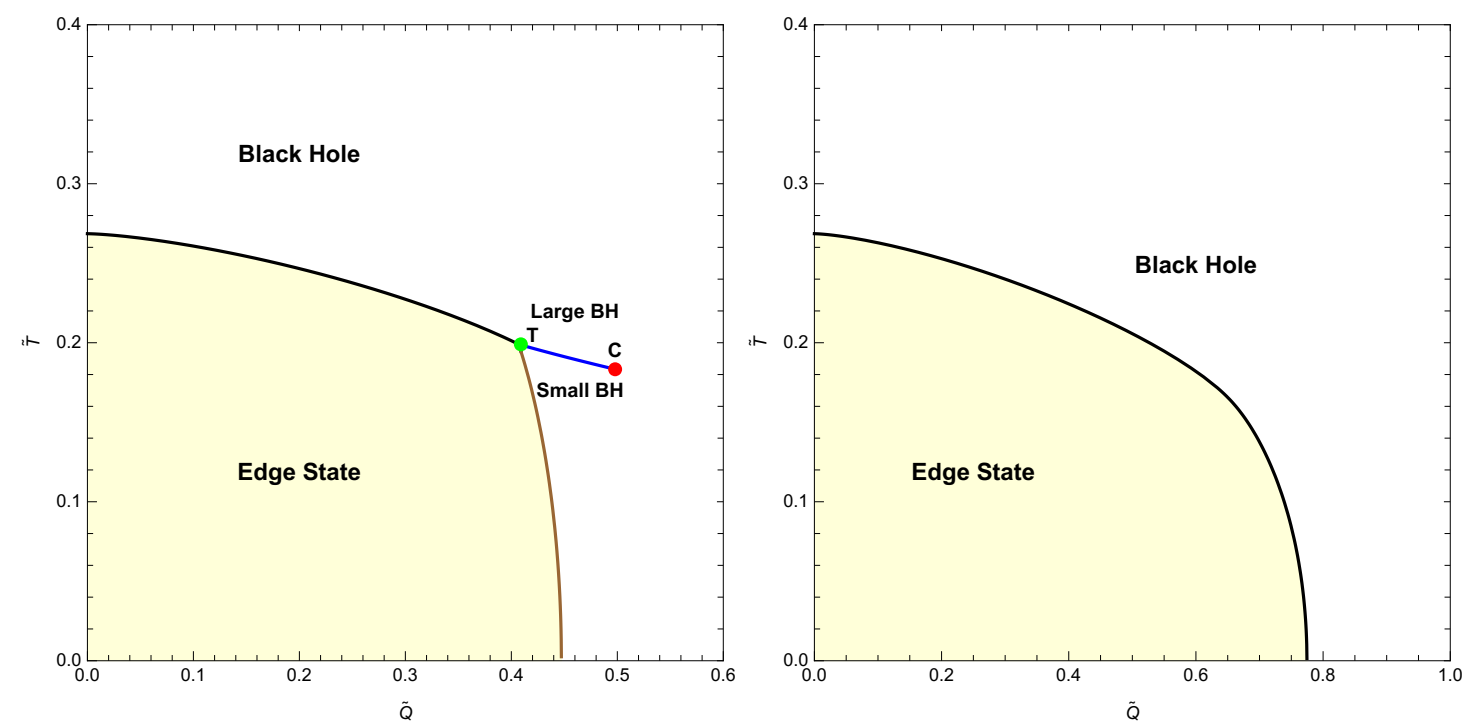

Figure 6. The phase diagrams in the $\tilde{Q}-\tilde{T}$ phase space. Left Panel: $\tilde{a}=0.05<\tilde{a}_{c}$. The firstorder phase transition line separating Large BH and Small BH is displayed by the blue line, and it terminates at the critical point, marked by the red dot and annotated by $\mathrm{C}$. There are also a first-order phase transition line between $\mathrm{BH}$ and the edge state, depicted by the black line, and a first-order phase transition line between SBH and the edge state, depicted by the brown line. These first-order phase transition lines merge together at the tricritical point, marked by the green dot and annotated by T. Right Panel: $\tilde{a}=0.15>\tilde{a}_{c}$. There is a first-order phase transition line separating $\mathrm{BH}$ and the edge state, displayed by the black line.

transition lines merging together at a tricritical point. At the critical temperature, there is a critical point, corresponding to a second-order phase transition, beyond which there is only one phase. For $\tilde{a}=0.15$, there is only one phase transition line, which separates the edge state from the black hole phase. Note that we only focus on spherical topology in our paper, so it is possible that there are some other states of lower free energy in a different topological sector with the same charge and temperature. If this happens, the stable phases discussed above are only metastable.

Using asymptotically AdS boundary conditions, the thermodynamics of BI black holes was considered in [68-70]. For the RN type in Regions I and II, the phase structure of a BI black hole in a cavity is analogous to that of the corresponding BI-AdS black hole. However for the Schwarzschild-like type in Regions III, IV and V, we find that there are some differences between the thermodynamics of the BI black holes under these two boundary conditions. For example, a LBH/SBH/LBH reentrant phase transition, which consists of a $\mathrm{LBH} / \mathrm{SBH}$ first-order phase transition and a $\mathrm{LBH} / \mathrm{SBH}$ zeroth-order phase transition, could occur for the BI-AdS black holes in Region V of [70]. On the other hand, such reentrant phase transition is not observed for any BI black hole in a cavity. Nevertheless, it is naive to claim that the phase structure of BI black holes depends on details of the boundary conditions. A crucial observation is that, if there were no edge states, the phase structure of the BI black hole in a cavity would be quite similar to that of the BI-AdS black hole. In fact, if the edge state is ignored, the inset of the bottom left 
panel in figure 5a shows that, as the temperature increases, there is a finite jump in free energy leading to a zeroth-order phase transition from Large $\mathrm{BH}$ to Small $\mathrm{BH}$, which is followed by a first-order phase transition returning to Large BH. This $\mathrm{LBH} / \mathrm{SBH} / \mathrm{LBH}$ transition is just the reentrant phase transition.

In asymptotically AdS spaces, the Euclidean action needs to be regulated to cancel the divergences coming from the asymptotic region. One can use the background-substraction method to regularize the Euclidean action by subtracting a contribution from a reference background. The reference background and the edge state play a similar role in determining the global minimum of the free energy. For a Schwarzschild-AdS black hole, the reference background is just the thermal flat space, which is the same as the edge state at $x=0$ for a Schwarzschild black hole in a cavity. As the temperature decreases, both the Schwarzschild black hole in a cavity and the Schwarzschild-AdS black hole thus experience the Hawking-Page transition to the thermal flat space [11]. Although there is ambiguity about the reference background of charged black holes, one can circumvent this by using the counterterm subtraction method [71, 72], in which the Euclidean action is regularized in a background-independent fashion by adding a series of boundary counterterms to the action. In $[68,70]$, the counterterm subtraction method was used to compute the Euclidean action for a BI-AdS black hole. For a RN black hole in a cavity, the global minimum of the free energy is never at the endpoints, which explains that the phase structures of the RN black hole in a cavity and the RN-AdS black hole have extensive similarities [14]. However for a BI black hole in a cavity, there are some regions in the $\tilde{Q}, \tilde{a}$ and $\tilde{T}$ parameter space, where the global minimum of the free energy is at $x=0$. Different phase structure from that of a BI-AdS black hole appears there. Our results imply that, in some region of the parameter space of the BI-AdS black hole, there might be other states of lower free energy with the same charge and temperature. Inspired by Schwarzschild-AdS black holes, one natural candidate is the thermal AdS space filled with charged particles. However, the backreaction of the charged particles on the AdS geometry should be considered, which could lead to formation of a naked singularity. It is inspiring to explore the possible equilibrium phases of lower free energy for charged AdS black holes.

\section{Acknowledgments}

We are grateful to Zheng Sun and Zhipeng Zhang for useful discussions and valuable comments. This work is supported in part by NSFC (Grant No. 11005016, 11175039 and 11375121). 
Open Access. This article is distributed under the terms of the Creative Commons Attribution License (CC-BY 4.0), which permits any use, distribution and reproduction in any medium, provided the original author(s) and source are credited.

\section{References}

[1] S.W. Hawking and D.N. Page, Thermodynamics of Black Holes in anti-de Sitter Space, Commun. Math. Phys. 87 (1983) 577 [INSPIRE].

[2] J.M. Maldacena, The Large $N$ limit of superconformal field theories and supergravity, Int. J. Theor. Phys. 38 (1999) 1113 [hep-th/9711200] [INSPIRE].

[3] S.S. Gubser, I.R. Klebanov and A.M. Polyakov, Gauge theory correlators from noncritical string theory, Phys. Lett. B 428 (1998) 105 [hep-th/9802109] [INSPIRE].

[4] E. Witten, Anti-de Sitter space and holography, Adv. Theor. Math. Phys. 2 (1998) 253 [hep-th/9802150] [INSPIRE].

[5] E. Witten, Anti-de Sitter space, thermal phase transition and confinement in gauge theories, Adv. Theor. Math. Phys. 2 (1998) 505 [hep-th/9803131] [INSPIRE].

[6] A. Chamblin, R. Emparan, C.V. Johnson and R.C. Myers, Charged AdS black holes and catastrophic holography, Phys. Rev. D 60 (1999) 064018 [hep-th/9902170] [INSPIRE].

[7] A. Chamblin, R. Emparan, C.V. Johnson and R.C. Myers, Holography, thermodynamics and fluctuations of charged AdS black holes, Phys. Rev. D 60 (1999) 104026 [hep-th/9904197] [INSPIRE].

[8] M.M. Caldarelli, G. Cognola and D. Klemm, Thermodynamics of Kerr-Newman-AdS black holes and conformal field theories, Class. Quant. Grav. 17 (2000) 399 [hep-th/9908022] [INSPIRE].

[9] R.-G. Cai, Gauss-Bonnet black holes in AdS spaces, Phys. Rev. D 65 (2002) 084014 [hep-th/0109133] [INSPIRE].

[10] D. Kubiznak and R.B. Mann, P-V criticality of charged AdS black holes, JHEP 07 (2012) 033 [arXiv: 1205.0559] [INSPIRE].

[11] J.W. York Jr., Black hole thermodynamics and the Euclidean Einstein action, Phys. Rev. D 33 (1986) 2092 [inSPIRE].

[12] H.W. Braden, J.D. Brown, B.F. Whiting and J.W. York Jr., Charged black hole in a grand canonical ensemble, Phys. Rev. D 42 (1990) 3376 [InSPIRE].

[13] S. Carlip and S. Vaidya, Phase transitions and critical behavior for charged black holes, Class. Quant. Grav. 20 (2003) 3827 [gr-qc/0306054] [INSPIRE].

[14] A.P. Lundgren, Charged black hole in a canonical ensemble, Phys. Rev. D 77 (2008) 044014 [gr-qc/0612119] [INSPIRE].

[15] J.X. Lu, S. Roy and Z. Xiao, Phase transitions and critical behavior of black branes in canonical ensemble, JHEP 01 (2011) 133 [arXiv:1010.2068] [INSPIRE].

[16] C. Wu, Z. Xiao and J. Xu, Bubbles and Black Branes in Grand Canonical Ensemble, Phys. Rev. D 85 (2012) 044009 [arXiv:1108.1347] [INSPIRE].

[17] J.X. Lu, R. Wei and J. Xu, The phase structure of black D1/D5 (F/NS5) system in canonical ensemble, JHEP 12 (2012) 012 [arXiv:1210.0708] [INSPIRE].

[18] J.X. Lu and R. Wei, Modulating the phase structure of black D6 branes in canonical ensemble, JHEP 04 (2013) 100 [arXiv: 1301.1780] [INSPIRE]. 
[19] D. Zhou and Z. Xiao, Phase structures of the black $D p-D(p+4)$-brane system in various ensembles I: thermal stability, JHEP 07 (2015) 134 [arXiv: 1502.00261] [INSPIRE].

[20] Z. Xiao and D. Zhou, Phase structures of the black Dp-D( $p+4)$-brane system in various ensembles II: electrical and thermodynamic stability, JHEP 09 (2015) 028 [arXiv: 1507.02088] [INSPIRE].

[21] P. Basu, C. Krishnan and P.N. Bala Subramanian, Hairy Black Holes in a Box, JHEP 11 (2016) 041 [arXiv: 1609. 01208] [inSPIRE].

[22] Y. Peng, B. Wang and Y. Liu, On the thermodynamics of the black hole and hairy black hole transitions in the asymptotically flat spacetime with a box, Eur. Phys. J. C 78 (2018) 176 [arXiv: 1708.01411] [INSPIRE].

[23] Y. Peng, Studies of a general flat space/boson star transition model in a box through a language similar to holographic superconductors, JHEP 07 (2017) 042 [arXiv:1705.08694] [INSPIRE].

[24] Y. Peng, Scalar field configurations supported by charged compact reflecting stars in a curved spacetime, Phys. Lett. B $\mathbf{7 8 0}$ (2018) 144 [arXiv:1801.02495] [INSPIRE].

[25] N. Sanchis-Gual, J.C. Degollado, P.J. Montero, J.A. Font and C. Herdeiro, Explosion and Final State of an Unstable Reissner-Nordström Black Hole, Phys. Rev. Lett. 116 (2016) 141101 [arXiv: 1512.05358] [INSPIRE].

[26] S.R. Dolan, S. Ponglertsakul and E. Winstanley, Stability of black holes in Einstein-charged scalar field theory in a cavity, Phys. Rev. D 92 (2015) 124047 [arXiv:1507.02156] [InSPIRE].

[27] S. Ponglertsakul, E. Winstanley and S.R. Dolan, Stability of gravitating charged-scalar solitons in a cavity, Phys. Rev. D 94 (2016) 024031 [arXiv:1604.01132] [INSPIRE].

[28] N. Sanchis-Gual, J.C. Degollado, C. Herdeiro, J.A. Font and P.J. Montero, Dynamical formation of a Reissner-Nordström black hole with scalar hair in a cavity, Phys. Rev. D 94 (2016) 044061 [arXiv: 1607.06304] [INSPIRE].

[29] S. Ponglertsakul and E. Winstanley, Effect of scalar field mass on gravitating charged scalar solitons and black holes in a cavity, Phys. Lett. B 764 (2017) 87 [arXiv:1610.00135] [INSPIRE].

[30] N. Sanchis-Gual, J.C. Degollado, J.A. Font, C. Herdeiro and E. Radu, Dynamical formation of a hairy black hole in a cavity from the decay of unstable solitons, Class. Quant. Grav. 34 (2017) 165001 [arXiv:1611.02441] [INSPIRE].

[31] O.J.C. Dias and R. Masachs, Charged black hole bombs in a Minkowski cavity, Class. Quant. Grav. 35 (2018) 184001 [arXiv:1801.10176] [INSPIRE].

[32] O.J.C. Dias and R. Masachs, Evading no-hair theorems: hairy black holes in a Minkowski box, Phys. Rev. D 97 (2018) 124030 [arXiv:1802.01603] [INSPIRE].

[33] F. Simovic and R. Mann, Critical Phenomena of Charged de Sitter Black Holes in Cavities, Class. Quant. Grav. 36 (2019) 014002 [arXiv:1807.11875] [INSPIRE].

[34] L. McGough, M. Mezei and H. Verlinde, Moving the CFT into the bulk with TT, JHEP 04 (2018) 010 [arXiv: 1611.03470] [INSPIRE].

[35] H.H. Soleng, Charged black points in general relativity coupled to the logarithmic U(1) gauge theory, Phys. Rev. D 52 (1995) 6178 [hep-th/9509033] [INSPIRE].

[36] H. Maeda, M. Hassaine and C. Martinez, Lovelock black holes with a nonlinear Maxwell field, Phys. Rev. D 79 (2009) 044012 [arXiv:0812.2038] [INSPIRE]. 
[37] S.H. Hendi, B. Eslam Panah, S. Panahiyan and A. Sheykhi, Dilatonic BTZ black holes with power-law field, Phys. Lett. B 767 (2017) 214 [arXiv:1703.03403] [INSPIRE].

[38] J. Tao, P. Wang and H. Yang, Testing holographic conjectures of complexity with Born-Infeld black holes, Eur. Phys. J. C 77 (2017) 817 [arXiv:1703.06297] [INSPIRE].

[39] X. Guo, P. Wang and H. Yang, Membrane Paradigm and Holographic DC Conductivity for Nonlinear Electrodynamics, Phys. Rev. D 98 (2018) 026021 [arXiv:1711.03298] [INSPIRE].

[40] B. Mu, P. Wang and H. Yang, Holographic DC Conductivity for a Power-law Maxwell Field, Eur. Phys. J. C 78 (2018) 1005 [arXiv:1711.06569] [inSPIRE].

[41] S.H. Hendi and M.H. Vahidinia, Extended phase space thermodynamics and P-V criticality of black holes with a nonlinear source, Phys. Rev. D 88 (2013) 084045 [arXiv:1212.6128] [INSPIRE].

[42] J.-X. Mo, G.-Q. Li and X.-B. Xu, Effects of power-law Maxwell field on the critical phenomena of higher dimensional dilaton black holes, Phys. Rev. D 93 (2016) 084041 [arXiv: 1601.05500] [INSPIRE].

[43] C.H. Nam, Non-linear charged dS black hole and its thermodynamics and phase transitions, Eur. Phys. J. C 78 (2018) 418 [InSPIRE].

[44] M. Dehghani, Thermodynamic properties of dilaton black holes with nonlinear electrodynamics, Phys. Rev. D 98 (2018) 044008 [INSPIRE].

[45] E. Ayon-Beato and A. Garcia, Regular black hole in general relativity coupled to nonlinear electrodynamics, Phys. Rev. Lett. 80 (1998) 5056 [gr-qc/9911046] [INSPIRE].

[46] E. Ayon-Beato and A. Garcia, New regular black hole solution from nonlinear electrodynamics, Phys. Lett. B 464 (1999) 25 [hep-th/9911174] [INSPIRE].

[47] K.A. Bronnikov, Regular magnetic black holes and monopoles from nonlinear electrodynamics, Phys. Rev. D 63 (2001) 044005 [gr-qc/0006014] [INSPIRE].

[48] M. Born and L. Infeld, Foundations of the new field theory, Proc. Roy. Soc. Lond. A 144 (1934) 425.

[49] T.K. Dey, Born-Infeld black holes in the presence of a cosmological constant, Phys. Lett. B 595 (2004) 484 [hep-th/0406169] [INSPIRE].

[50] R.-G. Cai, D.-W. Pang and A. Wang, Born-Infeld black holes in (A)dS spaces, Phys. Rev. D 70 (2004) 124034 [hep-th/0410158] [INSPIRE].

[51] S. Fernando and D. Krug, Charged black hole solutions in Einstein-Born-Infeld gravity with a cosmological constant, Gen. Rel. Grav. 35 (2003) 129 [hep-th/0306120] [INSPIRE].

[52] S. Fernando, Thermodynamics of Born-Infeld-anti-de Sitter black holes in the grand canonical ensemble, Phys. Rev. D 74 (2006) 104032 [hep-th/0608040] [InSPIRE].

[53] R. Banerjee, S. Ghosh and D. Roychowdhury, New type of phase transition in Reissner Nordström-AdS black hole and its thermodynamic geometry, Phys. Lett. B 696 (2011) 156 [arXiv: 1008.2644] [INSPIRE].

[54] R. Banerjee and D. Roychowdhury, Critical phenomena in Born-Infeld AdS black holes, Phys. Rev. D 85 (2012) 044040 [arXiv:1111.0147] [InSPIRE].

[55] A. Lala and D. Roychowdhury, Ehrenfest's scheme and thermodynamic geometry in Born-Infeld AdS black holes, Phys. Rev. D 86 (2012) 084027 [arXiv:1111.5991] [InSPIRE].

[56] R. Banerjee and D. Roychowdhury, Critical behavior of Born Infeld AdS black holes in higher dimensions, Phys. Rev. D 85 (2012) 104043 [arXiv:1203.0118] [INSPIRE]. 
[57] M. Azreg-Aïnou, Black hole thermodynamics: No inconsistency via the inclusion of the missing P-V terms, Phys. Rev. D 91 (2015) 064049 [arXiv: 1411.2386] [InSPIRE].

[58] S.H. Hendi, B. Eslam Panah and S. Panahiyan, Einstein-Born-Infeld-Massive Gravity: AdS-Black Hole Solutions and their Thermodynamical properties, JHEP 11 (2015) 157 [arXiv: 1508.01311] [INSPIRE].

[59] M. Kord Zangeneh, A. Dehyadegari, M.R. Mehdizadeh, B. Wang and A. Sheykhi, Thermodynamics, phase transitions and Ruppeiner geometry for Einstein-dilaton-Lifshitz black holes in the presence of Maxwell and Born-Infeld electrodynamics, Eur. Phys. J. C 77 (2017) 423 [arXiv: 1610.06352] [INSPIRE].

[60] X.-X. Zeng, X.-M. Liu and L.-F. Li, Phase structure of the Born-Infeld-anti-de Sitter black holes probed by non-local observables, Eur. Phys. J. C 76 (2016) 616 [arXiv:1601.01160] [INSPIRE].

[61] S. Li, H. Lü and H. Wei, Dyonic (A)dS Black Holes in Einstein-Born-Infeld Theory in Diverse Dimensions, JHEP 07 (2016) 004 [arXiv: 1606.02733] [INSPIRE].

[62] D.-C. Zou, S.-J. Zhang and B. Wang, Critical behavior of Born-Infeld AdS black holes in the extended phase space thermodynamics, Phys. Rev. D 89 (2014) 044002 [arXiv:1311.7299] [INSPIRE].

[63] S. Hossein Hendi, B. Eslam Panah, S. Panahiyan and M. Hassaine, BTZ dilatonic black holes coupled to Maxwell and Born-Infeld electrodynamics, Phys. Rev. D 98 (2018) 084006 [arXiv: 1712.04328] [INSPIRE].

[64] S.H. Hendi and M. Momennia, Reentrant phase transition of Born-Infeld-dilaton black holes, Eur. Phys. J. C 78 (2018) 800 [arXiv:1709.09039] [INSPIRE].

[65] B.R. Majhi and S. Samanta, P-V criticality of AdS black holes in a general framework, Phys. Lett. B 773 (2017) 203 [arXiv: 1609.06224] [INSPIRE].

[66] K. Bhattacharya and B.R. Majhi, Thermogeometric description of the van der Waals like phase transition in AdS black holes, Phys. Rev. D 95 (2017) 104024 [arXiv:1702.07174] [INSPIRE].

[67] K. Bhattacharya, B.R. Majhi and S. Samanta, Van der Waals criticality in AdS black holes: a phenomenological study, Phys. Rev. D 96 (2017) 084037 [arXiv:1709.02650] [INSPIRE].

[68] S. Gunasekaran, R.B. Mann and D. Kubiznak, Extended phase space thermodynamics for charged and rotating black holes and Born-Infeld vacuum polarization, JHEP 11 (2012) 110 [arXiv: 1208.6251] [INSPIRE].

[69] A. Dehyadegari and A. Sheykhi, Reentrant phase transition of Born-Infeld-AdS black holes, Phys. Rev. D 98 (2018) 024011 [arXiv:1711.01151] [INSPIRE].

[70] P. Wang, H. Wu and H. Yang, Thermodynamics and Phase Transitions of Nonlinear Electrodynamics Black Holes in an Extended Phase Space, JCAP 04 (2019) 052 [arXiv: 1808.04506] [INSPIRE].

[71] V. Balasubramanian and P. Kraus, A Stress tensor for Anti-de Sitter gravity, Commun. Math. Phys. 208 (1999) 413 [hep-th/9902121] [INSPIRE].

[72] R. Emparan, C.V. Johnson and R.C. Myers, Surface terms as counterterms in the AdS/CFT correspondence, Phys. Rev. D 60 (1999) 104001 [hep-th/9903238] [InSPIRE]. 